University of Wollongong

Research Online

Faculty of Informatics - Papers (Archive)

Faculty of Engineering and Information

Sciences

1995

\title{
Applications of smartcards for anonymous and verifiable databases
}

Thomas Hardjono

Jennifer Seberry

University of Wollongong, jennie@uow.edu.au

Follow this and additional works at: https://ro.uow.edu.au/infopapers

Part of the Physical Sciences and Mathematics Commons

\section{Recommended Citation}

Hardjono, Thomas and Seberry, Jennifer: Applications of smartcards for anonymous and verifiable databases 1995.

https://ro.uow.edu.au/infopapers/1115

Research Online is the open access institutional repository for the University of Wollongong. For further information contact the UOW Library: research-pubs@uow.edu.au 


\title{
Applications of smartcards for anonymous and verifiable databases
}

\begin{abstract}
In this paper we describe a practical solution towards anonymous and verifiable databases based on the use of smartcards and the recent Improved Leighton-Micali protocol for the distribution of keys. The scheme is addressed particularly to public data held in separate government databases with the aim of preventing unauthorized government institutions from gathering and merging private data concerning individuals from these separate containers. The solution can be realized through the recent Clipper Chip and smartcard technology, and its security relies on the strength of these technologies. The scheme is also extendible to mobile computing environments.
\end{abstract}

Disciplines

Physical Sciences and Mathematics

Publication Details

Thomas Hardjono and Jennifer Seberry, Applications of smartcards for anonymous and verifiable databases, Computers and Security, 14 (1995), 465-472 


\section{Applications of smartcards for anonymous and verifiable databases}

\section{Thomas Hardjono and Jennifer Seberry}

Centre for Computer Security Research, Department of Computer Science, University of Wollongong, Wollongong, NSW 2522, Australia

\begin{abstract}
In this paper we describe a practical solution towards anonymous and verifiable databases based on the use of smartcards and the recent Improved Leighton-Micali protocol for the distribution of keys. The scheme is addressed particularly to public data held in separate government databases with the aim of preventing unauthorized government institutions from gathering and merging private data concerning individuals from these separate containers. The solution can be realized through the recent Clipper Chip and smartcard technology, and its security relies on the strength of these technologies. The scheme is also extendible to mobile computing environments.
\end{abstract}

Keqwords: Security and protection, Database management, Network protocols.

\section{Introduction}

Security of public data represents an issue which $S$ is increasingly becoming important and relevant to all individuals within society. Public data can range from statistics which bear no direct impact on any given individual in society, to med- ical and financial information whose disclosure may affect an individual's standing within society. In traditional paper-based societies the gathering of such personal information concerning a particular individual was difficult to perform due to the shear amount of manual work involved. Hence, only certain government bodies could afford such data gathering based on some legal warrant.

In today's computerized world the collection and transfer of voluminous amounts of information over wide geographic distances has been accepted as a common everyday occurrence. With recent advances in fibre optics technology, the notion of superhighways for data is becoming a reality. With this increasing ease at which voluminous data can be transferred and the increasing speed of data processing systems, the capacity for data gathering and intelligent computerized processing has also been significantly increased. These advances, which are beneficial to society from one point of 


\section{T. Hardjono and J. Seberry/Applications of smartcards}

view, have raised questions from the opposite point of view, namely, of whether such computing power can be misused against society both by certain individuals within the society and by the very government upon which members of society have placed their trust. Accepting that for the functioning of a society some trust must be placed by the society on its government, a method of assurance must still be used to guarantee that an individual's personal details, which are spread across different government institutions, cannot be illegally gathered and merged together to give a total picture of that individual's private life.

One of the earliest efforts directed into finding possible solutions to this problem is that by Brandt et al. [1]. This effort recognized that databases belonging to different institutions must provide to the individual users the properties of the users being anonymous and the databases being verifiable. More specifically, when different data items are given by an individual to these distinct and separate institutions, these data items should not be identifiable by others as having come from the one same individual. The true identity of each individual must remain unknown to other individuals and to each institution. Each individual must also have the ability to verify that his or her personal details held by an institution are correct.

In the current work we investigate the issue of anonymous and verifiable databases in the context of recent developments in tamper-proof hardware, with the aim of presenting some practical solutions to the need of such databases. Our approach is founded on the use of smartcard technology coupled with an improved version of the recent Leighton-Micali key distribution protocol $[2,3]$. In the next section we discuss the motivations of the current work, with the aim of placing Brandt's work [1] in our context. This is followed in Section 3 by the description of the scheme, focusing on the Improved Leighton-Micali protocol and on the issues of anonymity, data storage and data verifiability. Section 4 closes with some brief remarks and conclusions.

\section{Motivations}

The need of a practical scheme to realize the notion of anonymous and verifiable databases is becoming self-evident in computerized nations. One recent example in Australia was the public debate over the Australian Identity Card [4] by which every Australian resident would be assigned a unique number as an identifying piece of information. This number would then be used as a pointer to cross-reference data in various government institutions which held information concerning the owner of the number. Although this move by the government was defeated, in actuality the government later proceeded to use the citizens' taxation file numbers more or less as a substitute for the proposed identity card.

One important recent development in the United States which has again brought the debate about citizens' right to privacy into the foreground is the introduction of the Clipper Chip [5, 6] and its related technology. The Clipper Chip is a highspeed and high-security encryption device to be used by the US Government for its telephone and other networking equipment. The chip has a classified encipherment algorithm and contains a secret key. Through a 'key-escrow' system an appointed government agency can obtain a legal warrant to wiretap communications between any two parties that are using the device. The main idea behind this notion is to provide secure communications to the users of the Chip against external attacks, while at the same time allowing the government to monitor communications that are suspected of being a threat to national security or to society in general (e.g. drug traffickers, industrial espionage).

This paper extends the notions embodied in the Clipper Chip concept towards another area, namely that of providing ways to achieve anonymous and verifiable databases. We require the appointed agency or authority to be a trusted adjudicator between the members of society and the other ordinary government institutions. In this 


\section{Computers \& Security, Vol. 14, No. 5}

way sensitive data concerning citizens in general may be guarded against illegal access while data concerning suspected citizens can be made readily available to the appointed authority. In the following discussion we will denote the appointed government agency as the Trusted Authority (TA). We assume that each institution holds a database containing every individual's details which are relevant to the functioning of the institution. Any exchange of data between departments must be through the Trusted Authority who regulates as to which details are exchangeable and who enforces the chosen policies. Thus, for example, the taxation department holds taxation-related information, while the health department has a health record of individuals that obtain medical service from the government hospitals. An individual is able to submit new details to each institution, respectively, and each individual can query each database independently without his or her identity being revealed.

Each individual has the duty to initially enrol himself or herself to the Trusted Authority, bringing their personal identification information (e.g. birth certificate, retina scan, fingerprint, DNA sequence). The Trusted Authority creates a pseudonym [1] for an individual corresponding to each institution that holds data about the individual. Hence, an individual has a different pseudonym when dealing with each institution. For each individual, the Trusted Authority issues a tamperproof smartcard containing that individual's set of pseudonyms and other cryptographic parameters. For a given institution, the Trusted Authority also issues cryptographic parameters which are stored in the tamper-proof smartcard belonging to an appointed trusted local authority (person) who is a representative of the institution (e.g. system administrator). Unlike the identity of individuals, each institution has a unique identity which is published.

The database at each institution is assumed to be managed by a trusted DBMS which can be used by staff members at the site only through a num- ber of tamper-free terminals [7, 8]. These tamperfree terminals represent the only valid access points to the database. A number of tamper-free terminals are also provided at the site for use by visiting individuals in the public, while remote tamper-free terminals may also be connected provided that a secure channel can be created between the remote tamper-free terminals and the local tamper-free terminals. The appointed representative for an institution has the duty to periodically load the cryptographic parameters from his or her smartcard to each of the resident tamperfree terminals at that institution. This configuration is shown in Fig. 1.

\section{Towards a practical scheme}

In this section we present a practical scheme for anonymous and verifiable databases based on the Improved Leighton-Micali (ILM) protocol [3]. The original Leighton-Micali protocol [2] had an inherent flaw which in our context allowed an attacker to read data belonging to an individual when it was in transit between the institution and the individual's terminal. This flaw has subsequently been solved and the protocol improved Zheng's work [3]. ${ }^{1}$

Following the requirements of $[2,3]$, we assume that tamper-proof VLSI chips are readily available to be incorporated into smartcards and tamperfree terminals. We also assume that a publicly known one-way hash function $h$ exists (which may also be replaced with a cryptographically strong pseudo-random function).

When an individual wishes to submit data to an institution or to verify existing data held by an institution, he or she must interact via a tamperfree terminal which establishes a connection with another tamper-free terminal located at the institution. Communications between these two terminals must be via a session key which is selected by either terminal and is transferred securely to

${ }^{1}$ The work of [3] has recently been published in [9]. 


\section{T. Hardjono and J. Seberry/Applications of smartcards}

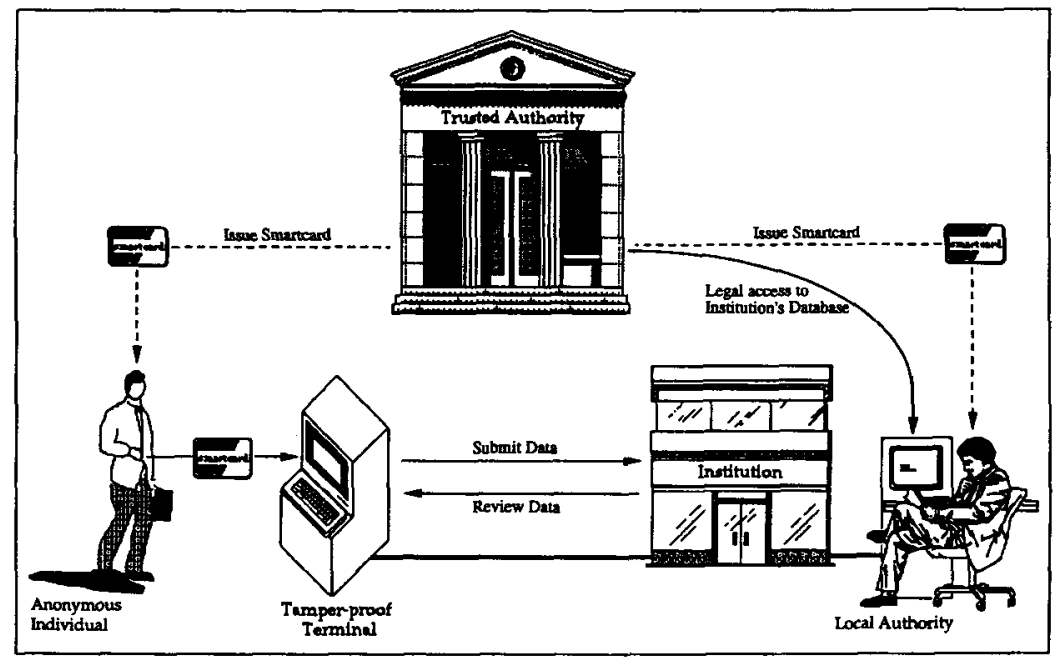

Fig. 1. Anonymous individuals and verifiable databases.

the other. The session key is then discarded by both terminals after the session is over. Newly submitted data is assumed to be placed in a temporary location within the institution's database to be read, verified and classified by one of the institution's staff members. Only then can such data be committed to the database. In the following, we assume that all communications are protected against replays (e.g. via timestamps or nonces).

\subsection{Session keys: the ILM protocol}

In the ILM protocol it is assumed that the Trusted Authority holds $M$ secret keys $\left(X_{1}, \ldots, X_{M}\right)$. Each secret key is chosen uniformly at random by the Trusted Authority and is of length $k$ bits. For each user $i$ who is enrolled into the system, the Trusted Authority selects $M$ random integers $\left(\alpha_{1}\right.$, $\left.\ldots, \alpha_{M}\right)$ from the interval $[1, L]$, where $L$ is an integer. ${ }^{2}$

The Trusted Authority then employs $h$ to compute $Y_{m}=h^{\alpha_{m}}\left(X_{m}\right)$ for all $m=1, \ldots, M$, where $h^{s}(X)$ indicates applying consecutively the func-

\footnotetext{
${ }^{2}$ Leighton and Micali recommended the size of $M$ be of the order $\mathrm{O}\left(B^{3} \log N\right)$, where $N$ is the number of users and $B$ is the upper-bound on the number of dishonest users $[2,9]$.
}

tion $h$ on an input $X$ for $s$ times. That is,

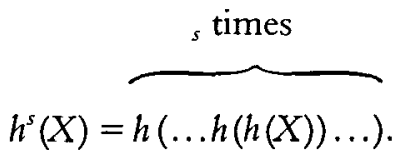

Here $\left(\alpha_{1}, \ldots, \alpha_{M}\right)$ becomes the public key of individual $i$ which is known to all institutions. The corresponding secret key $\left(Y_{1}, \ldots, Y_{M}\right)$ is then placed by the Trusted Authority into the tamperproof chip of the individual's smartcard. The smartcard is only delivered to the individual after the secret key has been inserted, and hence no person has access to the secret keys or the other secret parameters within that individual's tamperproof chip.

Assuming that individual $i$ needs to verify or submit data to institution $j$, then their respective terminals must establish a secure channel by way of encipherment using key $K_{i, j}$. This secure channel will afterwards be used to transfer the random session key $K_{s}$. The crucial requirement at this point is that both terminals must establish the same key $K_{i, j}$ independently without previous communications. This process can be done as follows 


\section{Computers \& Security, Vol. 14, No. 5}

[3]:

1. The terminal of individual $i$ must obtain the public key $\left(\beta_{1}, \ldots, \beta_{M}\right)$ of institution $j$. This public key can be resident within each tamperfree terminal or it can be read by the terminal from a publicly readable file.

2. After individual $i$ inserts his or her smartcard into the terminal, the terminal must provide the smartcard with the public key of the institution $j$. The tamper-proof chip within the smartcard of individual $i$ then computes the common key $K_{i, j}$ as:

$$
K_{i, j}= \begin{cases}h\left(h^{\delta_{1}}\left(X_{1}\right)\left\|h^{\delta_{M}}\left(X_{M}\right)\right\| i \| j\right), & i \leq j, \\ h\left(h^{\delta_{1}}\left(X_{1}\right)\left\|h^{\delta_{M}}\left(X_{M}\right)\right\| j \| i\right), & i>j,\end{cases}
$$

where $\delta_{m}=\max \left(\alpha_{m}, \beta_{m}\right), m=1, \ldots, M$ and |l denotes concatenation.

Note that the tamper-proof chip can easily compute $h^{\delta_{m}}\left(X_{m}\right)=h^{\left|\delta_{m}-x_{m}\right|}\left(Y_{m}\right)(m=1, \ldots, M)$ and thus $K_{i, j}$ because it has available the values $Y_{m}=h^{\alpha_{m}}\left(X_{m}\right)$ residing in its internal memory. The tamper-proof chip of the terminal at the institution performs symmetric procedures, and thus obtains the same key $K_{j, i}=K_{i, j}[3]$.

In order to aid our subsequent discussions we will simplify eq. (1) into

$K_{i, j}= \begin{cases}h(X\|i\| j), & i \leq j, \\ h(X\|j\| i), & i>j .\end{cases}$

As before, the $k$-bit value $X$ is chosen randomly by the Trusted Authority where $k$ should be sufficiently large, say $k \geq 100$, in order to prevent it from an exhaustive search attack [3]. The value $X$ is kept secret by the Trusted Authority, and during the enrolment of individuals the Trusted
Authority also injects a copy of $X$ into the chip of the smartcard belonging to the individual and into that belonging to the local authority at each institution. Hence, in fact, the value $X$ is common to all parties in the system.

\subsection{Anonymity}

In order to provide anonymity to individuals within the system, the Trusted Authority must create distinct pseudonyms for each user with respect to each of the institutions. In order to do this each individual $i$ must enrol in-person to the Trusted Authority and provide it with some identification information $P_{i}$. The Trusted Authority uniformly chooses a unique identity $I_{i}$ and a unique secret value $S_{i}$, and associates them with $P_{i}$. It is the duty of the Trusted Authority to keep the values $\left(P_{i}, I_{i}, S_{i}, S C_{i}\right)$ secure, where $S C_{i}$ is the unique chip number built into the tamperproof chip of the individual's smartcard. The same procedure is also observed for the local authority of each institution.

Assuming that each institution has been assigned a publicly known identity $B_{j}$, the Trusted Authority creates the pseudonym $I_{i, j}$ of the individual with identity $I_{i}$ with respect to $B_{j}$ as:

$I_{i, j}=h\left(S_{\mathrm{TA}}\left\|I_{i}\right\| B_{j}\right)$.

Here we assume that the encoding scheme for the identities of individuals $I_{i}$ and institutions $B_{j}$ is uniform. The key $S_{\mathrm{TA}}$ is maintained as secret by the Trusted Authority. The secret value $S_{i}$ and the pseudonyms for an individual are then inserted into that individual's chip. Similarly, each institution is given the respective pseudonym that the individual will present to the institution.

Another secret parameter injected into the tamper-proof chips of both the individual $I_{i, j}$ and the institution $B_{j}$ is a database key $D_{i, j}$, uniformly chosen by the Trusted Authority. This database key will be used to create other keys which are further used to control access to the database. Thus, for example, these created keys can be used 


\section{T. Hardjono and J. Seberry/Applications of smartcards}

to hide passwords of individuals, to encipher the access matrix or to encipher the data in the database. In this paper we will use them to encipher stored data, although it is clear to the reader that other modes of their usage are possible.

\subsection{Data storage}

Within each institution $B_{j}$ data in the database concerning individual $I_{i, j}$ must be stored in such a way that only the individual and the institution (i.e. its staff) can view the data. Assuming $R_{i, j}$ represents the data of individual $I_{i, j}$ at institution $B_{j}$, a key $K_{R_{i, j}}$ must be uniformly chosen by the local authority within the institution to be applied in order to hide data $R_{i, j}$. Bearing in mind that a secure DBMS running above a secure operating system is crucial for overall system security, there are a number of ways in which data can be stored in a manner that will make it accessible to users only through the key $K_{R_{i j}}$. One simple method through which data can be protected from unwanted disclosures is by way of direct encipherment using the above key $K_{R_{i, j}}$ (see [10]).

This encipherment key $K_{R_{i j}}$ must also reside in an enciphered form under a key which is available to the individual. This key-enciphering key is calculated by the staff's terminal at the institution as:

$$
K E_{i, j}= \begin{cases}h\left(X\left\|D_{i, j}\right\| I_{i, j} \| B_{j}\right), & I_{i, j} \leq B_{j}, \\ h\left(X\left\|D_{i, j}\right\| B_{j} \| I_{i, j}\right), & I_{i, j}>B_{j} .\end{cases}
$$

In addition, for each entry in the database belonging to the individual $I_{i, j}$ a signature or checksum $[11,12]$ must be created to prevent undetected changes to the data without the individual's consent. This is achieved by using the values $S_{i}$ and $S_{j}$ which are in the tamper-proof chips of the individual and the institution's local authority, respectively.

One simple way to create such signatures or checksums is as follows. When the individual is requested by the institution to verify and approve the data $R_{i, j}$ about the individual to be committed into the database, tamper-proof chips of the respective parties must generate certain parameters as input to some secure signature function sig. Thus, the chip belonging to the individual $I_{i, j}$ creates $t_{i, j}=h\left(R_{i, j}\left\|S_{i}\right\| B_{j}\right)$, while the chip belonging to the institution's local authority creates $t_{j, i}=h\left(R_{i, j}\left\|S_{j}\right\| I_{i, j}\right) \quad$ (note that here $\left.t_{i, j} \neq t_{j, i}\right)$. The two terminals onto which the individual and local authority are connected obtain the respective values from the chip within the respective (inserted) smartcards, and then the terminals exchange $t_{i, j}$ and $t_{j, i}$ over the secure channel established previously using the session key.

After receiving $t_{i, j}$ from the individual's terminal, the institution's terminal then computes the signature for the individual's entry. That is, the entry for individual $I_{i, j}$ within the database of institution $B_{j}$ is

$\left\{K_{R_{i, j}}\right\}_{K E_{i, j}},\left\{R_{i, j}\right\} K_{R_{i, j}}, \operatorname{sig}\left(R_{i, j}, t_{i, j}, t_{j, i}\right)$,

where the symbol ' \{\}$_{K}$ ' denotes encipherment using key $K$.

The institution's terminal then sends this complete entry (including the signature) and $R_{i, j}$ to the individual's terminal which re-computes the signature (if needed, the entry and the signature can also be sent by the individual's terminal to a lawyer who represents the individual). If both signatures are identical, the individual's terminal sends an acknowledgement to the terminal at the institution. Both terminals then erase the values $t_{i, j}$ and $t_{j, i}$. In this manner, neither the individual nor the institution can modify the data illegally, since neither $t_{i, j}$ nor $t_{j, i}$ are ever directly available to the individual or the institution's staff, respectively.

Here we have illustrated a simple method for the storage of data in an institution's database, focusing on the ease of access to the data from remote tamper-free terminals. Other secure data storage methods can be devised, and the reader is directed 


\section{Computers \& Security, Vol. 14, No. 5}

to [10-12] for a more comprehensive discussion on this particular issue.

\subsection{Verifiability}

When an individual $I_{i, j}$ wishes to view his or her data $R_{i, j}$ held at an institution $B_{j}$ the individual must use his or her smartcard with a tamper-free terminal:

1. The individual then selects via the tamper-free terminal the identity of the institution $B_{j}$ that holds the data the individual wishes to view.

2. After inserting his or her smartcard into the tamper-free terminal, the terminal provides the smartcard - and thus the chip within - with the identity $B_{j}$. The individual's terminal must also indicate to the institution's tamper-free terminal that a session is being requested. The institution's terminal then looks up the identity $I_{i, j}$ of the individual.

3. The individual's chip then computes $K_{i, j}$, while the chip within the institution's terminal computes $K_{j, i}$. As before, $K_{i, j}=K_{j, i}$.

4. The individual's terminal (or the institution's terminal) generates a session key $K_{s}$. The session key $K_{s}$ is then exchanged by way of enciphering it with $K_{i, j}=K_{j, i}$.

5. The institution's terminal then instructs the database system to return the entry $\left\{K_{R_{i, j}}\right\}_{K E_{i, j}}$, $\left\{R_{i, j}\right\}_{K R_{i, j}}, \operatorname{sig}\left(R_{i, j}, t_{i, j}, t_{j, i}\right)$ for individual $I_{i, j}$. This entry is enciphered using the session key $K_{s}$ and the result is dispatched to the individual's terminal.

6. The individual's terminal deciphers the entry using the session key $K_{s}$, and the key-enciphering key $K E_{i, j}$ is recreated following eq. (3). The individual's terminal then recovers $K_{R_{i, j}}$ and uses it to decipher and present to the individual the data $R_{i, j}$. The integrity of the data may also be verified by way of recreating the signature in the manner previously discussed. This would involve the institution's terminal re-computing $t_{j, i}$ and sending it to the individual's terminal via a secure channel.

\section{Remarks and conclusion}

In this paper we have extended the notions embodied in the Clipper Chip concept towards achieving anonymous and verifiable databases. The Trusted Authority creates a pseudonym for an individual corresponding to each institution that holds data about the individual. Hence, an individual has a different pseudonym when dealing with each institution. For each individual, the Trusted Authority issues a tamper-proof smartcard containing that individuals's set of pseudonyms and other cryptographic parameters. The database at each institution is assumed to be managed by a trusted DBMS which can be used by staff members at the site only through a number of tamper-free terminals. These tamper-free terminals represent the only valid access points to the database. A number of tamper-free terminals are also provided at the site for use by individuals in the public, while remote tamper-free terminals may also be connected, provided a secure channel can be created between the remote tamper-free terminals and the local tamper-free terminals. When an individual wishes to submit data to an institution or to verify existing data held by an institution, he or she must interact via a tamperfree terminal which establishes a connection with another tamper-free terminal located at the institution. Communications between these two terminals must be via a session key which is selected by either terminal and is transferred securely to the other. The session key is then discarded by both terminals after the session is over.

The security of the scheme relies on the tamperresistance of the chips and the randomness of the one-way hash function. To reduce the risk of abusing stolen chips, authentication of a chip's owner should be conducted by such means as user password [3]. In its current stage the scheme does not pretend to cover all possible points of 


\section{T. Hardjono and J. Seberry/Applications of smartcards}

attack, and clearly it does not provide a balanced burden of trust between an individual and an institution. After all, it is the institution that maintains the database containing the individual's private information. In practice it is difficult to prevent an institution from creating an informal and separate 'black list' database containing 'offthe-record' information upon which in reality it bases its decisions concerning a given individual. Other security measures are also required to prevent staff members of an institution from sharing data illegally with other institutions (e.g. manually copying onto a removable hard disk). The scheme in this paper represents a first step towards providing a practical mechanism in the face of an emerging new technology.

\section{Acknowledgements}

We thank Yuliang Zheng for bringing the ILM protocol to our attention. We also thank Azad Jiwa for comments and support. This work was supported in part by the Australian Research Council under the reference numbers A49130102, A9030136, A49131885 and A49232172.

\section{References}

[1] J. Brandt, I.B. Damgard and P. Landrock, Anonymous and verifiable registration in databases, in C.G. Gunther (ed.), Advances in Cryptology-Proc. EUROCRYPT '88 (Lecture Notes in Computer Science No. 330), Springer-
Verlag, 1988, pp. 167-176.

[2] T. Leighton and S. Micali, Secret-key agreement without public-key cryptography, in D.R. Stinson (ed.), Advances in Cryptology-Proc. Crypto '93 (Vol. 773 of Lecture Notes in Computer Science), Springer-Verlag, 1993, pp. 456-479.

[3] Y. Zheng, Amending Leighton and Micali's key distribution protocol, Technical Report Preprint 93-17, Centre for Computer Security Research, Computer Science Department, University of Wollongong, Sept. 1993.

[4] E. Smith, The Australia Card: the story of its defeat, Sun, South Melbourne, 1989.

[5] White House Press Release, The Clipper Chip initiative, IEEE Inf. Theory Soc. Newsl., 43(2) (1993) 21-23.

[6] DOC/NIST, A proposed Federal information processing standard for an escrowed encryption standard (EES), Fed. Reg., 58(145) (1993).

[7] J.K. Omura, A computer dial access system based on public-key techniques, IEEE Commun. Mag., 25(7) (1987) 73-79.

[8] P.J. Lee, Secure user access control for public networks, in J. Seberry and J. Pieprzyk (eds), Advances in Cryptology-Proc. AUSCRYPT '90 (Sydney) (Lecture Notes in Computer Science No. 453), Springer-Verlag, Jan. 1990, pp. 46-57.

[9] Y. Zheng, On key agreement protocols based on tamperproof hardware, Inf. Process. Lett., 53 (1995) 49-54.

[10] D.E. Denning, Field encryption and authentication, in D. Chaum (ed.), Advances in Cryptology: Proc. CRYPTO '83, Santa Barbara, CA, pp. 231-247, Plenum Press, New York, 1983.

[11] D.E. Denning, Cryptographic checksums for multilevel data security, in Proc. 1984 IEEE Symposium on Security and Privacy, Oakland, CA), IEEE Computer Society, April 1984, pp. 52-61.

[12] D.E. Denning, Commutative filters for reducing inference threats in multilevel database systems, in Proc. 1985 IEEE Symposium on Security and Privacy, Oakland, CA, IEEE Computer Scoeity, April 1985, pp. 134-146. 